\title{
UCRL- 11363
}

\section{University of California}

\section{Ernest $\mathrm{O}$. Lawrence Radiation Laboratory}

\section{POCKET-A POOR MAN'S LIBRARY SYSTEM FOR COMPUTER}

\author{
Berkeley, California
}




\section{DISCLAIMER}

This report was prepared as an account of work sponsored by an agency of the United States Government. Neither the United States Government nor any agency Thereof, nor any of their employees, makes any warranty, express or implied, or assumes any legal liability or responsibility for the accuracy, completeness, or usefulness of any information, apparatus, product, or process disclosed, or represents that its use would not infringe privately owned rights. Reference herein to any specific commercial product, process, or service by trade name, trademark, manufacturer, or otherwise does not necessarily constitute or imply its endorsement, recommendation, or favoring by the United States Government or any agency thereof. The views and opinions of authors expressed herein do not necessarily state or reflect those of the United States Government or any agency thereof. 


\section{DISCLAIMER}

Portions of this document may be illegible in electronic image products. Images are produced from the best available original document. 


\section{UNIVERSITY OF CAILIFORNIA \\ Lawrence Radiation Laboratory \\ Rerkeley, California}

AEC Contract No. W-7405-eng -48

POCKET - A POOR MAN'S LIBRARY SYSTEM FOR COMPUTER

Alan Natapoff

April 8, 1964 
POCKET $_{\nu}$ - A POOR MAN'S LIBRARY SYSTEM FOR COMPUTER

\author{
Alan Natapoff \\ Lawrence Radiation Laboratory \\ University of California \\ Berkeley, California
}

April 8, 1964

\begin{abstract}
This report describes a computer library system, POCKET, which was designed for general use with scientific experiments. POCKET is written entirely in Fortran IV programming language and can be applied to a wide variety of tasks by providing an optional, user-written subroutine. The program accepts and yields a variety of input and output forms, under the direction of the user. A versatile control-card reading routine permits the entry of arbitrary additional data. POCKET is designed to be convenient, versatile, and simple at the cost of speed. On a typical problem, it handled the input and output of about 1600 records per minute on the 7094 , using standard Fortran library routines. A simplicity of logic is achieved by imbedding the forms both of the library records and the editing instructions in the same form. The system has been in use for several years.
\end{abstract}




\section{POCKET - A POOR MAN'S LIBRARY (A MINIMUM FORTRAN PROGRAM)}

\section{INTRODUCTION}

A. Some Pseudo-philosophical Considerations (What Every Librarian Knows)

In Old Iestament days, taking a slave from among the Sons of the Covenant was a perilous affair. The laws governing their treatment were so severe that unless great care were exercised, the annoyance often outweighed the benefit.

In the view of certain computer users, a similar situation exists with respect to the use of computer programs to perform library functions. Yet, the prospect of having a computer assume the drudgery of record keeping is so attractive that it can cause or to forget warnings. There follows a dis cussion of some of the unignored warnings and of the particular compromise between generality and convenience embodied in the Fortran program POCKET, a program that pretends to deal with the problems in first order.

Let us think of a library system as a program that:

I. Summons all previously gathered information and presents it as an ordered entity,

II. Permits periodic augmentation of the body of information always preserving the order of the whole,

III. Permits the editing of information already digested.

These functions may be called the basic library functions. Most library programs, however, perform additional functions such as that of abstracting, (presenting selected subportions of the collection of information), scrutiny (examination of and rumination over pieces of new information as they enter the body of information), integration (comparison of several neighboring records in search of information not contained in any single record), and error checking.

Although the list of basic library functions might be rejected by many as incomplete, they already place heavy burdens on any program designed to implement them. The editing function, for example, implies a system of notation for referring to records already in the library.

The program must, of course, be able to distinguish editing references from the ordinary information being fed into the library. If editing and ordinary information are handled concurrently by the library (as convenience dictates they should be) either there must be separate forms for the presentation of each, or else the program must be told whether a given piece of information is to be treated as data or as edit information. To clarify this point a little, we can think of an editor in a publishing house who is given a typed manuscript to work on. He must insert pieces of copy at times, but he must also give instructions to his secretary as to how to type the new version. Further his secretary must be able to distinguish words 
that are to be entered into the manuscript from words that are meant only to instruct her in the revision of the manuscript. If she could not so distinguish, poems might be printed as

"Wherefore should any set thee love apart.?

Type this triple-spaced with $1-1 / 2$ inch margins

Seeing none but I makes much" of naught (He saïd)"

The editors, of course, have a simple solution. They write comments to their secretaries (editing information) in red pencil, and insertions in blue pencil. This provides a convenient double language adequate for editing. A possible alternate solution to the problem is to precede each sentence of an editing comment with the symbol $*$, and each sentence of an insertion with $\$$.

For example, of the two sentences below:

* Use your judgment in modifying the blocking of the text.

\$ Use your judgment in modifying the blocking of the test.

the first would be an editing comment, and the second an insertion into the text.

This latter scheme is presented because it is an exact analog of the system we actually use in our program, POCKET. It realizes the dual language necessary to permit editing, and it enables us to cast all statements to be fed into the computer in precisely the same form, with the first field, say, distinguishing data from editing instructions.

Thus we achieve our dual language by imbedding both sublanguages in a single language of higher level. Doing so grants us a certain technical simplicity.

To avoid serious complications analogous to those situations in logic related to Russell's Paradox, we must restrict the editing function of our. . program to operate only on information (not for example, on other editing instructions). This completes our introduction to the problem.

A few simple examples given later may dispel some of the haze introduced by the generalities presented.

\section{B. General Description}

The POCKET library system was designed for use with scientific experiments and claims to be simple, general, versatile and open-ended. It does not claim efficiency either of operation or of memory use. Using the POCKET guide; a person could tailor his own library program in one or two hours without needing to know anything technical about programming. The resulting program.would perform all of. what we have called the "library functions" (collection, collation, and updating) provided the basic data he wished to keep in his library satisfy a few simple requirements as to form.

be:

The basic unit of the library is the record. A record can in general 
machine.

i. A card (converted to magnetic tape) punched as on a keypunch netic tape).

ii. A group of such cards numbered consecutively (converted to mag-

iii. A binary record written by the computer on an earlier run.

The restrictions imposed by POCKET are:

i. Each reel of tape processed by the program must consist entirely of records of identical length and format. (The single relaxation permitted is discussed later in the section on the SHRIVE subroutine).

ii. Each record must contain fewer than 1024 pieces of information.

iii. Each record must contain three (preferably more) dummy fields for use by the program in implementing the "double language" spoken of in the introduction. These fields may each be located anywhere in the record, but they must carry no data. For example, if the record were a single puched card, leaving columns 73 through 80 blank on all cards would satisfy this requirement. (See the section on recommended procedures for further details).

\section{What POCKET Does}

The POCKET library system is a set of routines written entirely in FORTRAN IV language. Its input and output are managed by the standard Fortran library routines. It handles any subset of the following: library.

i, An input tape (or set of tapes) of new data to be entered into the

ii. An old library tape (or set of tapes) to be updated by the input.

iii. A new library tape to be written that contains the merged old library and new data.

Each of these consists entirely of records of a single format although they need not agree with each other. If, for example, any input were on singlecard records, the entire input would have to be on single-card records, but the output could be $\overline{\text { a tape }}$ of binary records.

Let us assume, for the moment, that all the "sources" are in use (input, old library, and new library). Then the program merges the input with the old library according to an ordering scheme specified by the user in the control cards, and writes a new library tape. The functions of the various parts of the system are best illuminated by giving the history of a record that is on the point of being written on the new library.

First, the record is examined by subroutine CENSOR to see if any editing is to be done on it. Perhaps the user has specified that this particular record is to be deleted. Perhaps the record is to be "suppressed" (see the description of CENSOR for a discussion of what this means). In either case, CENSOR makes the decision, and communicates it to the main program.

Second, the record is presented to the SHRIVE subroutine for whatever action is deemed appropriate. SHRIVE is a dummy subroutine that may be written by the user to achieve any function not provided for by the system. The SHRIVE routine has access to all the information available to the main program and is therefore expected to provide a medium for a very large range of applications. 
Third, the record is written on the new library, provided that CENSOR has not previously caused its deletion. This writing is achieved by the ESTHET routine.

Fourth and last, the record is examined by the BARD subroutine, which sees if the user has specified in the control cards that a summary of the record be written, and obeys.

These four functions comprise the heart of the system. CENSOR performs the editing; SHRIVE performs any task the user specifies; ESTHET mediates all input-output functions; BARD writes summaries of records, if they are requested. Each subsidiary subroutine is described in some detail in a later series of sections.

It is clear that the so-called "control cards" (cards punched by the user to guide the function of the system) play an important role in the system. We say a few words about them here and defer detailed description : to a special section and to the section on the SPADE subroutine.

For a very wide range of applications, the control cards are the only medium used to communicate with the system. Each card contains about six to ten pieces of information, and the information is used for several major purposes.

First, the control cards describe the records appearing on each source (input, old library, new library), specify which sources are in use, and give the information needed to actually run the program (numbers of tape reels, tape unit numbers, etc. ).

Second, the control cards give information to be used by the various subsidiary subroutines. They specify which optional functions are to be performed and provide the parameters needed to perform them.

Third and last; the control cards permit entry of arbitrary sets of. information into the memory for use, if needed, with the SHRIVE routine. With this facility, a user need not provide any programming, but can enter his parametric data conveniently when he provides the information needed by the program itself.

The table below lists the major and minor subroutines, with a short description of the function of each. For each routine, there is a separate discussion in a later section. For descriptions of the less important routines, the user is referred to the FORTRAN source listings themselves. These contain extensive comments to facilitate their use. 
Table of the major and minor subroutines in the POCKET system

\begin{tabular}{|c|c|}
\hline Subroutine & Function \\
\hline POCKET & Central routine \\
\hline BARD & $\begin{array}{l}\text { Writes descriptions of selected records, when requested to } \\
\text { do so by user through the control cards }\end{array}$ \\
\hline CENSOR & Controls all editing functions (deletion, suppression) \\
\hline ESTHET & $\begin{array}{l}\text { Cares for all input and output of records from and to the } \\
\text { various sources }\end{array}$ \\
\hline ETSEQ & Sees to the options that order the input records when requested \\
\hline SPADE & Controls handling of the control cards \\
\hline TENDRE & $\begin{array}{l}\text { Tends to the ending of output reels and the detection of the } \\
\text { ends of input reels }\end{array}$ \\
\hline 'BLEDA & $\begin{array}{l}\text { Block data program storing certain fixed information in } \\
\text { storage before execution starts }\end{array}$ \\
\hline CIFIE KA & $\begin{array}{l}\text { Decides whether a given tape number is in use by the monitor } \\
\text { system }\end{array}$ \\
\hline CHOPIN & $\begin{array}{l}\text { Places a given alphabetic ( } B C D \text { ) text in an array convenient } \\
\text { for writing }\end{array}$ \\
\hline CIT & Initializes certain regions of storage \\
\hline CLOCHE & Oversees the use of the clock-interrupt feature \\
\hline CONUBI & $\begin{array}{l}\text { Converts integers from, one base to another. (Used to con- } \\
\text { vert from } B C D \text { to integer form) }\end{array}$ \\
\hline DERERA & $\begin{array}{l}\text { Called by ESTHET for input-output control. This is the only } \\
\text { routine that actually calls input or output routines for handling } \\
\text { library records. }\end{array}$ \\
\hline EREXIT & $\begin{array}{l}\text { Causes an error exit of the program in case of palpable } \\
\text { malfunction. Gives a memory dump and an error message. }\end{array}$ \\
\hline EXIT & Causes a dump when EXEM causes a Fortran error exit \\
\hline IDIOM & Inserts single digits into numbers written to any base \\
\hline JUGGLE. & $\begin{array}{l}\text { Subroutine of ESTHET permitting arbitrary manipulations of } \\
\text { records }\end{array}$ \\
\hline OPINE & Handles all on-line comments to operators \\
\hline REWUNL & Rewinds and unloads a given tape unit \\
\hline RIVAL & $\begin{array}{l}\text { Mediates comparisons between two records to determine } \\
\text { precedence, identity, or similarity }\end{array}$ \\
\hline SPARE & $\begin{array}{l}\text { Calls input-output routines for SPADE: (DERERA: ESTHET:: } \\
\text { SPARE: SPADE) }\end{array}$ \\
\hline SUTTEE & Sets up the writing of record summaries for BARD \\
\hline
\end{tabular}


TRAP Senses the condition of the clock for clock-interrupt options ZENO Searches arrays logarithmically for table look-up purposes. Used with subroutine CENSOR to scan editing entries. 


\section{CONTROL CARDS FOR POCKET LIBRARY SYSTEM}

\section{A. Introduction}

The control cards for the POCKET library system form the tool for shaping the system to the needs of each particular problem. They are supposed to be simple and convenient to use. They perform their function by filling certain lists in COMMON storage that are referred to by the system. When these lists are completed, the program is ready to execute the library program tailored by them.

Since the control cards refer to several separate lists, it is convenient to divide them into sets, each set specifying a particular list. The card marking the end of each set also specifies the list to which the next set refers. This will be made explicit after the form of the control card has been discussed.

\section{B. Description}

Each control card is divided into three parts which are, in order, data, card number, mask. The data is the information to be read into the list, and is separated into a number of fields, each field filling one element of the list being filled. The card number, if positive, indicates into which places in the list the data from the card are to be inserted. If the card number is negative, the card is called a "marker card"., which is not considered to contain data but rather causes the program to stop reading cards. Specifically, if the card number on a card is $-N$ ( $N$ positive), then reading ceases. For $N=1$ or. $N=2$, the program modifies its own parameters and begins to read again as discussed in the section on subroutine SPADE. For $\mathrm{N}=3,4$, or 5, an error return will occur. $N=6$ marks the end of a set of cards, but implies that another set is to follow. The digits of the mask determine which list the next set will fill. In particular, 10XMASK(9) + MASK(10) gives the number of the list (see below) which will be filled by the next set of control cards. $N=7$ marks the end of a set of cards and implies that the current set is the last of the current block and that another block is to follow. For $\mathrm{N}=8$ or more, the implication is that we have completed reading all: the sets for the last block of the current run. The division of the program into blocks is discussed in separate section.

In general then, positive control-card numbers indicate where information is to be stored. Negative control-card numbers indicate "marker cards". Subsets of data for a given pass are separated by a card numbered -6, sets of data for successive passes are separated by a card numbered -7 , and the end of the data for the last pass of a run is marked by a card numbered -8 or less.

The mask is a set of ten integers used on cards of positive card. number for cutting and fitting data of different format into a single array. This feature, however, is never needed for the standard options of the POCKET library. It is provided in case the user-written SHRIVE routine requires a mixed array. The section on the SPADE subroutine contains a complete description of its use. Ordinarily, the mask digits on data cards are left blank. On cards having negative card numbers (marker cards), the mask serves an entirely different purpose. The digits in it are used to control the 
further course of the program. In particular, if the card number is -6 , the last two digits of the mask (see above) form the number of the list that is to be filled by the succeeding set of cards:: The use of the mask for cards numbered -1 and -2 is discussed in the section on subroutine SPADE.

\section{The Lists and Their Function}

There is provision for 12 lists, of which five, (JINN, KHYBER, JORDAN, FORM, LOBOS), are usually required, two more are required for the special case of multi-record inputs (see the section on subroutine ESTHET), one is left blank for use as a common working storage area, and four provided for optional data used in connection with the users SHRIVE routine. The lists are given in order below, each preceded by its identifying index:

\section{JINN (General Input Quantities)}

JINN is the major array to be filled. The first control cards read in for any pass are assumed to refer to JINN. The control cards are assumed to be in the standard format number 1, an integer form (10I6, 4X I6, 10I1). The standard formats for reading (10I6, 4X I6, 10I1), (5E12.4, 4X I6, 10I1), $(10 \mathrm{~A} 6,4 \mathrm{X} \mathrm{I6}, 10 \mathrm{I} 1)$, and $(5012,4 \mathrm{X} \mathrm{I6}, 10 \mathrm{I1})$ and the corresponding standard formats for writing for later offline printing are given in subroutine's CIT and BLEDA. One major function of the array is provision of operating parameters for the various major subroutines.

Equivalent to the area

\begin{tabular}{|c|c|c|c|}
\hline Name of array & from JINN( ) : & $\cdots$ to JINN( ) & Pertinent routine \\
\hline MUSSET & 11 & 20 & BARD \\
\hline LENOX & 37 & 40 & ETSEQ \\
\hline KENO & 51 & 60 & CENSOR, TENDRE \\
\hline MURAD & 61 & 70 & ESTHET \\
\hline LOREN & 71 & 80 & CENSOR \\
\hline
\end{tabular}

Outside of the routines named these parts of JINN are referred to if at all, only to write out their contents.

JINN(1) "LUHSUN" contains the logical number of the tape unit onto which all writing for off-line BCD printing of remarks, error messages, control card contents, etc., is done.

$\operatorname{JINN}(2,3)$ contains the logical number of the tape unit on which record reports, if any, requested via BARD will be written. If the report is for a record just read from the (input, old library); it will be written on tape LUHSUN $(2,3)$.

JINN $(21,22,23)$ "LASER" contains the number of words on a record from or to the (input, old library, new library).

JINN $(24,25,26)$ "JUNO"' contains the logical number of the tape unit on which the (input, old library, new library) reel is to be mounted: 
JINN $(31,32,33)$ "JAFFA" contains the number of tape reels expected to be fed into the (input, old library, new library).

JINN (41) "KLAXON" is the maximum number of records to be written on any single output reel.

JINN (42) "NUECES" is the number of back records to be kept in storage after being written on the new library. The records are escalated in ANNALS. and are available for examination by SHRIVE. This feature is useful in facilitating "integration of records", the extraction from a set of neighboring records of information that was not contained in any single record. In particular, different measurements of the same experiment may be compared and conflicts in interpretation resolved. This feature, called the escalating feature, is discussed more fully in a separate section.

JINN $(43,44,45)$ "LANUM", "LADEN", "NIBBLE". In order to use storage efficiently, all storage of record references is kept together in ANNALS. This region is consecrated to two different purposes, however-the escalation of events and the storage of editing references. The portion of ANNALS devoted to each is determined by LANUM, LADEN, and NIBBLE. First, NIBBLE is the length of bank in storage to be set aside for each record. Four such banks are set aside in ANNALS for ordinary processing. Of the remainder, the fraction LANUM/LADEN is devoted to escalation, and the rest to storing the editing commands to be applied during execution of the program.

JINN (46) "JERKIN" (end-reel suppression code). At the end of a reel of the new library, a POCKET-type end-of-reel record is written. This is a record, recognized by TENDRE, that indicates that the end of the reel has been reached, but does not abort the program as would an end-of-file record might. This POCKET-type end-of-reel record is not written on the new library if JERKIN is an odd number.

JINN $(48,49,50)$ "LOTHAR" give the tape-handling code for the (input, old library, new library). The tape-handling code is a three-digit binary number whose bits refer, in order (low-order bit first), to (ending file, rewinding, unloading). If the appropriate binary digit is $(0,1)$, the corresponding function for the appropriate source (is not, is) suppressed if it would have been performed. For example, suppose LOTTHAR(1) $=6=(110)_{2}$. Then the ending of file is not suppressed, but rewinding and unloading a re suppressed for the input [source 1 is referred to by LOTHAR(1)]. Now the input reels are never written on, so that no end-file would be written in any case. Thus, the input reel is left precisely where it was. This may be convenient for use in the next pass.

The general rules for tape handling are:

System tapes are never rewound or unloaded, nor are ends-of-file written on them. The minor subroutine CHEKA tells which tapes are system tapes.

Input tapes are never written on nor are ends-of-file written on them.

Otherwise, all tapes are ended (file), rewound, and unloaded unless specified othe rwis'e: by the :appropiria te entry: iin LOTHAR. 


\section{2, 3, 4, 5. CERES, GUISE, ICARUS, and LUCRE}

Optional arrays p. rovid.ed.. for use with user-written SHRIVE routine, if needed.

6. KHYBER Code specifying the kind of data in each word of the record

KHYBER(J) is $(1,2,3,4)$ according as the information stored in the $\mathrm{Jth}$ word of the record is of (Integer, Floating-point, Alphabetic; Octal) mode. It need not be true that input, old library, and new library have records that correspond in the order of items: For example; the record.written on the new library may be longer than the input record. Or, the items on the input records may appear in a different order on the old-library records. Such circumstances occur, for example, when the POCKET system isised to change the form of a library to another, perhaps more convenient, form. Apart: from use in SHRIVE, the array KHYBER is used to facilitate comparison of input and old library records so that the order of records is preserved in the new library. The array refers to the single form of record appearing in memory while POCKET itself is handling the record. Differences of order and length such as those discussed above, are accommodated by the ESTHET routine. The means of accommodation is explained in the section discussing that routine. Thus, there.is one form of record in memory at a time, ir respective of the disparities in forms of input and output, and KHYBER refers to this single form. The disparities are resolved in ESTHET.

\section{JORDAN (Order specification)}

Specifying the order of records in our library reduces to the problem of specifying how to decide which of two given records $A$ and $B$ is to precede the other. The decision is made by comparing the contents of A and $B$ for $a$ specified set of fields, in order, until an unequal pair occurs. The set of fields to be compared is given in JORDAN. The number of the first field to be compared is given (by the control cards) in JORDAN (1) and the nth in JORDAN (n). The program compares the fields of records $A$ and $B \bar{a}$ s prescribed by JORDAN until, for some $n, I=J O R D A N(n)$ and $A(I)$ is not equal to $B(I)$. If $A(I)$ is greater or less than $B(I)$, then $(B ; A)$ is said to precede the other. Since numbers of fields must be positive integers, we specify the length of the list in JORDAN by a zero or a negative element. If JORDAN $(J)$ is ( 0 , negative) it implies then that the last element. of JORDAN is the $(\mathrm{J}-1, \mathrm{~J})$ th (see the example below). If the JORDAN list is exhausted before an unequal pair A(I), B(I) is found, the record from the old library is assumed (by right of seniority) to precede the record from the input.

For example, if JÖRDAN is $(8,6,3,-7)$, then $A(8)$ is compared to $B(8)$. If $A(8)$ is greater than $B(8)$, $B$ goes on the new library first, and vice versa. For $A(8)=B(8), A(6)$ is compared to $B(6)$ in the same way. If all four pairs of elements are equal $[A(8)=B(8), A(6)=B(6), A(3)=B(3)$, and $A(7)=B(7)]$, then whichever of $A, B$ came from the old library will go first on the new library.

\section{EREWON (Working Storage)}

EREWON is working storage for all routines and is treated as erasable. No control card should be provided. 


\section{FORM (Format Stätements)}

FORM provides formats for BCD records of input and output and for record reports to be written by. BARD. Starting with a left parenthesis ( and ending with a right parenthesis), whole FORTRAN formats are read in and stored in FORM. An index to the meaning and usage of these formats is given in the array LOBOSS (location of beginning of statement). The only restriction on the storage of these formats in FORM is that each new format statement must start in a new computer word (no computer word may contain parts of two format statements).

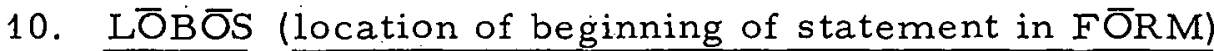

Every BCD record that is to be read or written by POCKET must have its format specified. In particular, this holds true for records read from or into the (input, old library, new library) and record reports written about records of the (input, old library, new library). Because of the three-part division of both of these functions, there is a corresponding division of the LOBOS a rray that implemients them. LOBOS is assumed to be divided into three sections of MACRO words each (where MACRO is specified by the BLEDA-CIT routines): "These sections are reasonably large (50 locations long in one version of POCKET) to accommodate the multi-card record forms of data discussed in the section on the ESTHET routine. For multi-card records; location 1 of each block contains the address of uniform format used for all multi-card records on first reading. Location 2 contains the address for the card numbered 0 and location $n+2$, for card numbered $n$. MUSSET(1) (see section on subroutine BARD) contains the corresponding address in each block for the formats to be used for record reports. The magnitude of MUSSET(1) is presumed to be greater than (maximum card number) +2 and

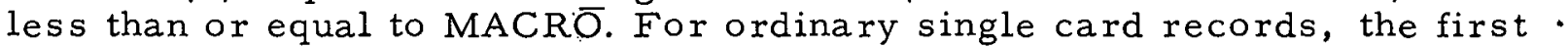
location contains the location of "the format".

11. MAFIA (number of fields)

MAFIA helps specify the structure of multi-card records. It is automatically filled in by the program for simple records (not of multi-card form). MAFIA(n+2) contains the number of fields on the card numbered n. MAFIA(1) contains the number of fields on the uniform format for all such cards.

\section{INGRAM (integrated MAFIA)}

INGRAM $(n+2)$ contains the location in the record into which the first field of card number $\mathrm{n}$ is to be read. If no entries are read in by control cards, the program assumes the entries to be successive according to card number. For single record format, too, no entries are needed; the program provides them during initialization.

\section{OSchematic Description of la Typical Set of Control Cards}

In the list below, a double pair of parentheses surrounding the name of an array, e.g. ((JINN)), refers to a set of cards of positive record number that will fill that array. A single set of parentheses surrounding a pair of numbers (NCARD, NEXT) refers to a single card whose record number is 
NCARD and whose columns 79 through 80 contain the number NEXT, the index of the array to be filled by the next set of control cards.

$((\operatorname{JINN})),(-6,6),((\operatorname{KHYBER})),(-6,7)((\operatorname{JORDAN})),(-6,9),((\operatorname{FORM})),(-6,10)$, ((LOBOS)), (-6,2), ((CERES)), (-8, ISSW)

A detailed example of a set of control cards is given with the description of the SHRIVE routine.

The last entry ( -8 , ISSW) means that, where in each earlier entry the last group of ten columns of the control card (columns 71 through 80 ) contains the single number $6,7,9,9,10,0$ r 2 , respectively, in this case the ten columns fill the ten locations ISSW(1) through ISSW(10). These parameters are interpreted by the prögram as. follows:

ISSW(1) is interpreted as a three-bit binary number equal to $I 1+2 \times I 2+4 \times I 4$, where we have $((I 1, I 2, I 4))=(0,1)$ if a $(n)$ ((input, old library, new library)) (is not, is) to be read or written on the current pass. These parameters are used in POCKET.

ISSW(2) equals $I 1+2 \times I 2+4 \times I 4$, where $((I 1, I 2, I 4))=(0,1)$ if the ((input, old library, new library)) (is not, is) in binary form. This information is used in ESTHET.

ISSW (3) equals $I 1+2 \times I 2+4 \times I 4$, where $((I 1, I 2, I 4))=(0,1)$ according as the ((input, old library, new library)) (is not, is) in multirecord format. An input source is : ultirecord format if several numbered cards are used instead of a single unnumbered card. This information is used by the ESTHET routine.

ISSW(6) equals I $+2 \times I 2+4 X \cdot I 4$, where $((I 1, I 2))=(0,1)$ if the records of the ((input, old library)) (are not, are) to be ordered, and $I 4=(0.1)$ if full ordering. (is not, is) to take place. The partial-ordering options remove any records that would precede, according to the specified ordering scheme, the previous record read from the same source. If full ordering is specified, the records so removed (and written on an auxiliary tape) are merged with the new library when the source from which they were taken is exhausted. This information is used by the ETSEQ routine. 


\section{SUBROUTINE SPADE}

\section{A. Introduction}

SPADE is a flexible routine that handles the control cards. The three major aspects to its function are reading, writing for off-line printing, and writing for off-line punching. Reading is the means of entering information conveniently; writing for off-line printing permits, among other things, summary and presentation of the data read in, for the convenience of the user; writing for off-line punching facilitates, among other things, restoration of memory after an interruption of the program.

\section{B. Calling sequence}

\section{CALL SPADE}

(MODE, NTREAD, NTRITE, NTPUN, LENGTH, IRATE, INDEX, MERROR, LABEL, BUFFER, MASK, ARRAY, FORMAT, INFORM)

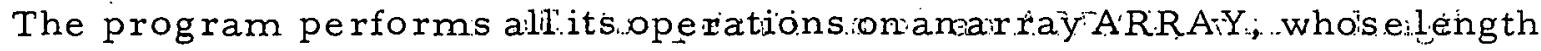
is assumed to be LENGTH. The type of information stored in the array is indicated by INDEX. Formats for the input-output operations are assumed to be stored in the array FORMAT. These formats are further described by the information in the array INFORM. The (reading, writing for off-line printing, writing for off-line punching) is done on or from tape unit number (NTREAD, NTRITE, NTPUN). When writing is done for off-line printing, the BCD characters stored in LABEL are used to label the array. When reading is done, reading of control records terminates when a card having a negative:record number is encountered. The absolute value of that record number is stored in IRATE on return. Should an obvious error occur in the execution of the routine, a return to the calling program occurs with MERROR unequal to zero. All reading proceeds through a buffer array BUFFER that always contains the contents of the last control record read. Of the various functions that can be performed by the routine, the particular subset to be performed on a given entry is specified by MODE.

\section{Dictionary of calling sequence variables}

\section{1. $\mathrm{MODE}$}

MODE, a coded integer specifying the subset of functions to be performed during the current entry, is equal to $M 1+3 \times M 3+9 \times M 9+27 \times M 27+81 \times M 81$. It is treated by the program as a five-digit ternary number whose digits (M1, M3, M9, M27, M81) specify the operation of the five major options (reading, writing for off-line printing, writing for off-line punching, recycling, format insertion). In each case; the digit equal to $(0,1,2)$ means that the corresponding option is ignored, partially invoked, or fully invoked. Partial invocation is the same as full invocation for all options except the second, writing for off-line printing. In that case, partial invocation causes headings to be omitted in the printing. 
2, 3, 4. NTREAD, NTRITE, and NTPUN

NTREAD, NTRITE, and NTPUN are the variable tape numbers of the units to be used for reading, writing for off-line printing, and writing for off-line punching. For example, if NTREAD. is 2, and reading is to be done, then the reading is done from the tape unit that the system calls number 2 . (In some systems, IBSYS for instance, this need not be tape unit. 2. The system may look up the number of the actual unit to be used in a table.)

\section{LENGTH}

LENGTH is the number. of words in the array being processed. For writing for off-line printing or punching, LENGTH tells the number of words to be printed or punched. For reading, it specifies a maximum size array into which one may read. If the program is told to read a piece of information into a location outside this specified region, an error return to the calling program occurs.

\section{IRATE}

IRATE is the index of a record causing termination of reading. When reading is being performed, it continues until a record is read that has a negative record number, $-N$. IRATE is set equal to $N$.

\section{INDEX}

INDEX indicates formats to be used in reading and writing during the current entry. If INDEX is 1 , then the format stored starting in [FORMAT $(1, I, 1)$, FORMAT $(1, I, 2)]$ will be used for [ reading, writing for later off-line punching or writing for later off-line printing]. The POCKET system adopts the convention that $I=(1,2,3,4)$ refers to $(I, E ; A, O)$ type formats. $I=(5,6, \ldots, 10)$ are reserved for optional formats employed by the user through the formatinsertion option (see below). The actual formats used for each index are prescribed by the block-data subroutine BLEDA (or the CIT subroutine on machines where no block-data subroutines exist). See the section on the BLEDA-CIT routines for further discussion.

\section{MERROR}

MERROR indicates errors. If an obvious error occurs during the execution of SPADE, MERROR is set equal to a nonzero value that indicates the nature of the malfunction, and an immediate return to the calling program follows. If no error occurred, MERROR is zero on exit. The error causing an error return can be deduced from MERROR by examining the listing of the SPADE routine.

\section{LABEL}

LABEL is one BCD word label attached to the array when written out for later off-line printing. 


\section{BUFFER}

BUFFER is a one-dimensional array in memory through which all read-in data passes before being inserted into ARRAY. The contents of the field on the record terminating the last reading cycle are in BUFFER upon return to the calling program.

\section{MASK}

MASK is a one-dimensional array in memory into which all suppression masks are read. The suppression mask on the record terminating the last reading cycle is in MASK upon return to the calling program. The function of the suppression mask is discussed below.

\section{ARRAY}

ARRAY gives the location in storage of the first word of the array on which the routine is to operate.

\section{FORMA T}

FORMAT is the three-dimensional array containing the input and output formats to be used by the routine. The Ith reading (and writing for offline punching) format is stored starting in location FORMAT $(1, I, 1)$, while the Ith format to be used for writing for off-line printing istarts: in. . FORMAT $(1, I, \overline{2})$. These regions are assumed to have been filled before entry. In the POCKET system, this filling is accomplished in the BLEDA and CIT routines.

\section{INFORM}

INFORM is an array of information describing the formats stored in FORMAT. INFORM (1,I) equals number of words of information in a record of type I. INFORM (2,I) is the number of words in the suppression mask of a record of type I. INFORM $(3, \mathrm{I})$ is a two-letter BCD code indicating the general form of data read in using a format of type $I$. This code is printed out when the writing for off-line-printing option is invoked. For example, the letters bI ( $b=b l a n k)$ might indicate that the format is of integer type.

\section{Description}

SPADE has five major functions of which, on each entry, it performs some subset. It examines each function to see. if it is to be performed on the current entry, then performs or ignores it accordingly. The subset of functions to be performed is specified, as stated above, by the argument MODE. The major functions are listed and described in order below.

\section{Reading}

The program starts reading records from the tape unit specified by NTREAD, and according to the INDEXth reading format in FORMAT. Each record is assumed to be composed, in order, of a set of data fields, a record number, and a set of mask fields. These àre read into BUFFER, JCARD, and MASK respectively. There are assumed to be NFIELD = INFORM (1, INDEX) data fields on the record and NMASK=INFORM (2, INDEX) mask fields. 
If JCARD is nonnegative, the information is transferred from BUFFER to the appropriate part of ARRAY, subject to the suppression mask whose operation is described below. The appropriate part of ARRAY is simply the part that is the (JCARD+1)th group of NFIELD locations. For JCARD =0, for example, the fields are transferred from BUFFER to ARRAY(1), ARRAY(2),... ARRAY(NFIELD). For JCARD = 7, the transfer takes place to locations ARRAY (7 X NFIELD + 1), . ARRAY (7XNFIELD + NFIELD). In general, BUFFER is transferred to the NFIELD locations of ARRAY starting with ARRAY (JCARD XNFIELD+1). For JCARD XNFIELD $\geqslant$ LENGTH, an error return to the calling program occurs.

The suppression mask simply suppresses the transfer of a word from BUFFER to its proper position in to ARRAY if the corresponding word in MASK is 1. Such a suppression leaves the position in ARRAY unchanged from its previous value. If, for example, a suppression mask has ten elements $(1,0,0,1,7,7,3,5,6,0)$ in the same order, and there are five fields of data on that record whose record number is 1, then the following occurs. The elements ARRAY (6) through ARRAY (10) are referred to by record number 1 and are stored in BUFFER (1) through BUFFER (5). After the transfers are complete, we find

$$
\begin{aligned}
\text { ARRAY }(6) & =\text { unchanged from previous value } \\
(7) & =\text { BUFFER }(2) \\
(8) & =\text { BUFFER }(3) \\
(9) & =\text { unchanged from previous value } \\
(10) & =\text { BUFFER }(5)
\end{aligned}
$$

$$
\begin{aligned}
& \operatorname{MASK}(1)=1 \\
& \operatorname{MASK}(2)=0 \\
& \operatorname{MASK}(3)=0 \\
& \operatorname{MASK}(4)=1 \\
& \operatorname{MASK}(5)=7
\end{aligned}
$$

Uses for this suppress mask are discussed in the remarks. If JCARD is negative, then IRATE is. set equal to the absolute value of JCARD, reading is terminated immediately, and the program goes on to see if writing for offline printing is to be done.

As an exarruple of such a record as might be handled, we consider the Ith format $(5 E 12.4,4 X I 6,10 I 1)$ with $\operatorname{INFORM}(1, I)=5$, INFORM $(2, I)=10$, INFORM $(3, I)=2 \mathrm{HbE}$. This record has five data fields (cols. $1-12,13-24$, 25-36, 37,48, 49-60), a card number (cols. 65-70) and a mask of ten digits (cols. $71-80$ ).

\section{Writing for Later Off-line Printing}

The program writes the first LENGTH elements of ARRAY on the tape unit specified by (NTRITE) according to the INDEXth writing format in FORMAT [starting at FORMAT (2, INDEX)], with heading LABEL, INFORM (2, INDEX) words per record (line).

\section{Writing for Later Off-line Punching}

The program writes the first LENGTH elements of ARRAY on the tape unit specified by (NTPUN) according to the INDEXth reading format in FORMAT [starting at FORMAT (1, INDEX)], INF $\overline{O R M ~(1, ~ I N D E X) ~ w o r d s ~ p e r ~}$ record. These records are of the precise-form that can be read in by the program. This feature is useful therefore, in implementing interrupt-resume options in large programs. 


\section{Recycling}

If the recycle option is enabled (by MODE) and if a reading option is terminated by a record numbered -1 , then the program changes certain of its arguments and begins execution from the beginning at SPADE, as though SPADE had just been called with the changed parameters as arguments. The parameters changed are MODE, INDEX, and LENGTH, and their new values are taken from the mask on the record that caused the invocation of the option. In particular, the new value for the parameters are

$$
\operatorname{LENGTH}=10000 \times \operatorname{MASK}(1)+1000 \times \operatorname{MASK}(2)+100 \times \operatorname{MASK}(3)+10 \times \operatorname{MASK}(4)
$$

$$
+\operatorname{MASK}(5)
$$

unless the right-hand side is zero, in which case the previous value of LENGTH is retained:

$$
\begin{aligned}
& \operatorname{MODE}=100 \times \operatorname{MASK}(6)+10 \times \operatorname{MASK}(7)+\operatorname{MASK}(8) \\
& \operatorname{INDEX}=10 \times \operatorname{MASK}(9)+\operatorname{MASK}(10) .
\end{aligned}
$$

In short, if the ten-digit mask is LLLLLMMMII, then the first five digits form the new LENGTH (if nonzero), the next three give the new MODE, and the last two, the new index.

\section{Format Insertion (and Recycling with Parameters Unchanged)}

If the format insertion is enabled (by MODE) and if a reading option is terminated by a record numbered -2 , the program assumes that the data fields of that record contain, in BCD form, a format statement that is to be inserted into the array FORM.

The ten-digit mask UUUMMNNJII is used to convey the index to be attached to the new format (II), the number, NFIELD, of data fields on a record written according to the new format (NN), and the number, NMASK, of mask fields on a record (MM). The digit $J$ equals $(1,2)$ according as the format is the (reading, writing) format for the index I. If the new index is $I$, then JOFFRE (1,I) equals NFIELD and JOFFRE $(2, I)$ equals NMASK after the operation of this option. The program recycles (with arguments unchanged) after completing a format insertion, permitting immediate use of the new format.

As an example, suppose we wish to be able to read records of the format $(8 \mathrm{~A} \cdot 8, \mathrm{I} 6,10 \mathrm{I1})$ and that these records are to be written out for offline printing according to the format, including headings, (1X A9, 1H(I8, 1H) 8A8). We assume that the first eight fields of the first format are data fields, the next field, the card number, and the last ten fields, the mask. Thus we have NFIELD $=8$ and NMASK $=10$. This insertion could be achieved, MODE permitting, by the records:

(8A8, I6, 1.0I1) 
on will refer toithe förmats just inserted), and column 78 contains 1 (or 2), indicating that the card contains the reading (or printing) format. A second example appears in the section on the SHRIVE routine.

To be able to read the record above, SPADE must be operating under a $\mathrm{BCD}$ format on entry. In the POCKET system, for example, INDEX $=3$ provides a format suitable for the purpose (10A6, 4XI6, 10I1). The example above assumes that this. format was specified on entry for INDEX $=3$.

\section{E. Example}

Suppose we wished to read BCD records from tape 2 into the array CERES having 250 locations, and write out the contents of that array afterwards on tape 3 for later off-line printing. Suppose too, that the format according to which records were to be read or written is stored in [FORM $(1, I, 1), \operatorname{FORM}(1, I, 2)]$ and that the information describing these formats is stored in array JOFFRE. Assume further that arrays VAL and ISS:W are available for use as buffer and mask areas, respectively, and that the absolute value of the record number on the record-terminating reading is to be stored in KEY. Let MEAN be a location set aside to hold an érror code if needed, and JADE be a location holding the BCD name of the array for heading the printed output. Assume that no writing for off-line punching is to be done, that recycling is to be permitted if a record numbered - 1 is encountered, but that no format insertion is anticipated. A suitable calling sequence would then be

CALL SPADE $(62,2,3,0,250, K E Y, I, M E A N, J A D E, V A L, I S S W, C E R E S, F O R M, J O F F R E)$.

\section{F. Remarks}

The SPADE routine is designed to permit handling arrays in a versatile manner. In particular, any array may be built up from appropriate records on a single entry to the program by. use of suppression masks and the recycle option. This is true no matter what the types of data are contained in the array, what mixture occurs, what alternation occurs, or what mode of representing the data is most convenient. By cutting and masking, any array can be fabricated. SPADE is useful for achieving convenient dumps of memory in suitable formats and for preserving the contents of arrays for later restoration (both the preservation and restoration are performed by SPADE). 


\section{SUBROUTINE CENSOR}

\section{A. Introduction}

CENSOR mediates all editing functions of the POCKET library. There are two such functions, deletion and suppression, both of which operate on records of the old library. There is no editing feature applicable to records of the input. If desired, one may edit the input tapes by using a SHRIVE routine. Or, more conveniently, the tapes to be edited can be treated as old library tapes.

Deletion simply causes omission of records appearing on the old library from: the new library. "Suppression" is a procedure of marking records in a flexible way so that a suitably written SHRIVE routine can recognize the new status of the record. The word "suppression" is meant to indicate one possible use for the feature. Suppose a record becomes obsolete for some reason. It might be more convenient to mark it and ignore it thereafter than to delete it and fear that some day its information will be needed. The suppression might also indicate the completion of an action or: the correction of an error. The essential point is that it is much more convenient to perform a suppression than it is to enter a new record and the first function can often replace the second.

To suppress a record, then, is to insert an appropiriate number into it. Since suppression is achieved in a manner much like deletion, it is clas sified as an editing function and handled by the CENSOR routine.

\section{B. Calling Sequence}

The calling sequence is

CALL CENSOR (KODE, INVOKE, KATO, QUARRY).

CENSOR records or performs an editing task, according to KODE, on the record QUARRY. Information as to the current specifications for the editing of series of records is stored in the array INVOKE. KATO is a parameter not presently used.

$$
\text { C. Dictionary of Calling Sequence Variables }
$$

\section{1. $\underline{\mathrm{KODE}}$}

KODE checks the COMMON variable MOSQUE to find out the phase of POCKET then being executed. If MOSQUE is 3 (event-by-event or execution phase), KODE is examined. If $\mathrm{KODE}$ is $(1,2)$, the program (enters the editing instruction specified by the record QUARRY into the editing file, 'examines'. the record in QUARRY to see if it should be deleted or suppressed).

\section{INVOKE}

INVOKE is a dimensioned $(2,5)$ array of integers, usually made to correspond, through the calling sequence, to the COMMON array ISTACO. $\operatorname{INVOKE}(J, 1)$, INVOKE $(J, 2)$ refer, respectively, to the delete and suppress option. For $\mathrm{J}=1$, the element is 1 or 2 according as the current record is not $o r$ is to be edited, respectively. For $J=2$, the element is 1 or 2 
according as a series of editing references has not or has been invoked, respectively. For example, INVOKE $(1,2)=2$ means that the current single record is to be suppressed. INVOKE $(2,1)=1$ means that we are not currently in the midst of a series of records to be deleted.

$\operatorname{INVOKE}(I, 5)$ is used with the series editing feature (see below). INVIOKE $(I, 5)$ is the number of the editing entry now in core that invoked the last change in series status for the Ith type of edit function. $I=1,2$ corresponds to delete, suppress. The sign of INVOKE $(I, 5)$ is + or - according as the change enabled or disabled the status, respectively. For example, $\operatorname{INVOKE}(1,5)=-18$ means that the last change in the series delete status took place with reference to edit reference number 18 in core and that the reference caused a disabling of successive deletions.

\section{KATO:}

KATO is unused.

4. QUARRY

QUARRY is the record currently being processed through SHRIVE.

\section{Description}

CENSOR performs two major functions. First, it sees to the accumulation in storage of editing references as they occur on the input tape. Second, given a record, it searches storage for editing references to it. If it finds one, it proceeds accordingly

\section{Accumulation of Editing References ( $\mathrm{KODE}=1$ )}

Whenever POCKET finds a record whose MANDARth element $\left[\right.$ MANDAR $\left.=\operatorname{KENO}(2)^{\prime}=\operatorname{JINN}(52)\right]$ is not zero, it calls on $\overline{C E N S O R}$ to perform the task specified by the actual value, KANTOR, of that element. CENSOR proceeds by taking a subset of the fields of that command record and storing this subset in memory for later reference. The subset may contain as many as eight elements. The numbers of the elements to be chosen for the subset should be placed, in order, in LOREN(1), LOREN(2)... [that is JINN(71), $\operatorname{JINN}(72), \ldots]$. The number of such elements is stored in $\operatorname{LEER}=\operatorname{LOREN}(10)=\operatorname{JINN}(80)$.

Of the subset that may be chosen, the first two are required and fixed. They are, in order, the ordinal and the command. Thus, supposing that the ordinal field on the old library were the 15 th and the command field, the 13 th, $\operatorname{LOREN}(1) \doteq 15$ and $\operatorname{LOREN}(2)=13$. LOREN $(2)$ usually equals $K E N O(2)$, and LOREN(1) equals KALI = JINN(30). The difference is this. POCKET calls . CENSOR when the KENO(2)th field of a record is nonzero, but CENSOR uses the LOREN(2)th field as the command. Similarly, the ESTHET program numbers the new library in the KALIth word, but CENSOR refers to records according to the number in the LOREN(1)th word.

For deletion; only two parameters are needed--one to distinguish deletion from other functions (the command), and one to tell which record to delete. The greater versatility of the suppression function requires a larger 
number of parameters. There are two ways to specify a suppression, direct and indirect. Both follow the same plan. Both involve the contents of a suppress field (the NFth field of the record), a divisor (ND), a base (NB), and an insertion (NI). The process can be described as follows: The contents of the NFth field are divided by ND giving a quotient $M Q$ and a remainder MR. The first digit of MR, expressed to the base NB is changed from its old value to NI. The resulting new number MQA is multiplied by ND and the original remainder MR, restored. That is:

Then

$$
\begin{aligned}
C(N F) & =N D \times M Q+M R \\
M Q A & =M Q-M Q \text { modulo } N B+N I \text { modulo NB. }
\end{aligned}
$$

$$
\mathrm{C}(\mathrm{NF}) \doteqdot \mathrm{ND} \times \mathrm{MQA}+\mathrm{MR}
$$

For example, suppose we wished to indicate on the 27 th word of certain records that each had been found to be similar to some earlier record, within limits ranging in stringency from 0 to 16 on some appropriate scale. Suppose too, that we had earlier marked certain events as obsolete by setting the last bit of the suppression word equal to 1 . To avoid overlap, we leave " the first bit of the 27 th word unchanged by dividing by two before making any insertion. In this case, we have $N F=27, N D=2, N B=16$, and $N I=$ some number from 0 to 15 . Supposing that a particular event has the number 477 in its suppression word (number 27) before, and that we wish to indicate a stringency of 6 . The new value of the 27 th word is computed as follows:

$$
\begin{aligned}
& \mathrm{C}(27) \text { before suppression of event }=477=2 \times 238+1 \\
& \begin{aligned}
\mathrm{MQA} & =238-238 \text { modulo } 16+6 \text { modulo } 16 \\
& =238-14+6=230
\end{aligned} \\
& \mathrm{C}(27) \text { after suppression of event }=2.230+1=461 .
\end{aligned}
$$

Thus, we have indicated a change in one kind of status of the event, left the other kinds unchanged and used our memory space efficiently by permitting the storage of several pieces of information in a single word.

Four parameters are central to this process. They are called NF, ND, NB, and NI above. There are two ways of specifying them: First; rigidily for all events to be suppressed in the run; secondly, flexibly, by giving one or more of the parameters on the command card. In the rigid scheme, we have $\operatorname{KENO}(3,8,9,10)=\operatorname{JINN}(53,58,59,60)=(N F, N B, N D, N I)$, respectively. In the flexible scheme, the parameters are not specified by the control cards (as they are in the rigid scheme), but are specified in the command cards themselves, in fields specified by the control cards. In particular, $\operatorname{KENO}(4,5,6,7)=\operatorname{JINN}(54,55,56,57)$ contains the number of the word in the command phrase that contains (NF, NB, ND, NI). The command phrase is the set of words abstracted from the command record and stored in memory. This command phrase is the set, in order, of the LOREN(1)st, LOREN(2)nd,.. LOREN (LEER)th words of the record stored in the 1st, 2nd,..., LEERth words of the command phrase. Thus, the command phrase is built up from the words specified in LOREN and the suppress parameters (NF, NB, ND, NI). Further, when no indirect parameter is specified, the direct parameter, if any, is assumed. 
For example, suppose we want to implement the kind of suppression discussed above, that is, $N F=27, N D=2, N B=16$, and $N I$, a variable whose value ranges from 0 to 15 , is specified in field number 45 of the command record and is stored in word number 3 of the command phrase. Suppose, therefore, that we need only three words in our command phrase (LEER = 3). It is assumed that the LEER + 1stword of the command entry of NILE words is the number of times the command has been referred to. Lastly, assume that the second field of the record contains the number of the record, and that the forth field contains the command. We can achieve this by the following assignments:

\begin{tabular}{|c|c|c|}
\hline LOREN(1) & $=2$ & $\begin{array}{l}\text { (Record number field is the second field on the record and } \\
\text { is stored first in the command phrase.) }\end{array}$ \\
\hline LOREN(2) & $=. .4$ & $\begin{array}{l}\text { (Command field is the fourth field on the record and is } \\
\text { stored second in the command phrase.) }\end{array}$ \\
\hline LOREN(3) & $=45$ & $\begin{array}{l}\text { (The } 45 \text { th field on the record is stored third in the com- } \\
\text { mand phrase.) }\end{array}$ \\
\hline LOREN(9) & $=.4$ & $\begin{array}{l}\text { (NILE: we choose to leave four words in storage for each } \\
\text { command. Any number LEER }+1 \text { or greater will do.) }\end{array}$ \\
\hline LOREN(10) & $=\therefore 3$ & (LEER: there are three words in the command phrase.) \\
\hline $\mathrm{KENO}(1)$ & $=16$ & $\begin{array}{l}\text { (Assume the end-of-reel indication used by TENDRE is } \\
\text { located in the 16th word of the record.) }\end{array}$ \\
\hline KENO(2) & $=4$ & (The fourth field of the record contains the command.) \\
\hline KENO(3) & $=27$. & $\begin{array}{l}\text { (The } 27 \text { th field of each record is the one to contain the } \\
\text { suppress code } N F=27 . \text { ) }\end{array}$ \\
\hline $\mathrm{KENO}(4)$ & $=0$ & $\begin{array}{l}\text { [The number of the word in the command phrase that con- } \\
\text { tains the number of the field into which the suppress code } \\
\text { is inserted is } 0 \text {. That is, there is no indirect specification } \\
\text { of NF by a field on the record. NF is fixed at the value } \\
\text { given by KENO(3).] }\end{array}$ \\
\hline $\mathrm{KENO}(5)$ & $=0$ & $\begin{array}{l}\text { [There is no indirectly specified base. NB is fixed at a } \\
\text { value given by KENO(8).] }\end{array}$ \\
\hline $\mathrm{KENO}(6)$ & $=0$ & $\begin{array}{l}\text { [There is no indirectly specified divisor: ND is fixed at } \\
\text { value given by KENO(9).] }\end{array}$ \\
\hline $\mathrm{KENO}(7)$ & $=$ & $\begin{array}{l}\text { (The third field of the command phrase contains NI, the } \\
\text { numerical insertion for suppression:) }\end{array}$ \\
\hline KENO(8) & $=16$ & $(\mathrm{NB}=16)$ \\
\hline KENO(9) & 2 & $(N D=2)$ \\
\hline KENO(10) & $=$ & $\begin{array}{l}\text { (The insertion NI is specified indirectly, so this entry is } \\
\text { always ignored, irrespective of its value.) }\end{array}$ \\
\hline
\end{tabular}




\section{Search and Use (KODE $=2)$}

The file of edit references is searched using subroutine ZENO, and appropriate action is taken.

\section{E. The Command Code}

We have seen what deletion and suppression mean, we have noted. that they are specified by command cards (that is, cards whose command fields are not equal to zero), but we have not found out how to construct a. . command that will do our bidding. First, note that each command card may delete or suppress, but not both. Further, for either deletion or suppression, all commands refer to records by their ordinal numbers. These ordinal numbers.are provided by the ESTHET routine. No editing can be done on records lacking numbers, so that it may be necessary to run through POCKET once to insert them. Second; each delete or suppress reference is made to a single recordinone of three possible ways, characterized by the following descriptive phrases:

- 0 . Delete (or suppress) record number $\mathrm{N}$.

1. Delete (or suppress) the series of records starting with record number $\mathrm{N}$.

2. Delete (or suppress) the series of records ending with record number $N$.

The first.type of editing reference is self-explanatory. The second and third occur in pairs. The following restrictions must be observed in the use of editing references:

1. The editing records, appearing on the input tape, must appear in order of ascending $N$, where $N$ is the number of record in the the old library referred to by the edit reference.

2. Each edit reference of type 1 must be paired with a reference of type 2 that follows it with no intervening references other than of type 0.

3. No two edit references may contain the same value $N$.

Now we specify the construction of the command code, a two-digit ternary number $3 \times N 3+N 1$, where $N 1=(0,1,2)$ means that the record has (no, a delete, a suppress) reference. $N 3=(0,1,2)$ means that the type (see above) of edit is $(0,1,2)$; that is, the reference is to a (single, beginning of a series of, end of a series of) record(s). For example, a command of $7(=2 \times 3+1)$ on a record, $R$, means that the deletion, specified by an earlier command, of a series of records is to end with the record whose number is given on record R. Similarly, the command 0 specifies that no editing whatever is to be: done, and the command 2, that a single record is to be suppressed. 


\section{V. -SUBROUTINE SHRIVE - SHRILL VERSION}

\section{A. Introduction}

This is the user-written subroutine that performs any desired function not already available from the main program. All information in COMMON storage is available to the SHRIVE routine. In particular, all data read in from control cards is at the disposal of the routine, which is entered once for every record that is to be written on the new library. This entry occurs just before the writing is to take place.

\section{B. Calling Sequence}

The calling sequence is CALL SHRIVE (NODE, LEX, LAW, KHEDIV).

\section{Dictionary of Calling Sequence Variables}

1. NODE (not used).

2. $L, E X$

LEX is a four-digit ternary number, coded by SHRIVE to indicate to POCKET certain instructions about the processing of the event SHRIVE has just examined (that is, the event in QUIRK). Its composition is.

where

$\mathrm{LEX}=\mathrm{L} 1+3 \times \mathrm{L} 3+9 \times \mathrm{L} 9+27 \times \mathrm{L} 27$,

L1 is $(0,1,2)$ if the event just examined (is, is not, is not) to be written out on the new library.

L3 is $(0,1,2)$ according as (nothing, the pass, the run) is to be terminated.

L9 is $(0,1,2)$ according as the record just examined (is not, is, is) to be treated as though it were an end-of-reel record.

L27 is $(0,1,2)$ according as a POCKET-type end-or-reel record (is, is not, is not) to be written at the end of the pass. (This value of L27 should be specified by SHRIVE during its terminal entry.) The value of LEX is never changed by any program but SHRIVE.

3. LAW

$\overline{\mathrm{LAW}}$ controls the operation of BARD.: See the section on the BARD routine.

4. KHEDIV (Not used)

D. Description of a Typical SHRIVE Routine

SHRIVE is a user-written dummy subroutine which provides a flexible tool for the shaping of the POCKET library system to the user's needs. It is entered under five different circumstances distinguished by the value of the COMMON variable MOSQUE.

MOSQUE $=(1,2,3,4,5)$ corresponds to the function (initialize, resume, execute, interrupt, terminate). The user should test the variable MOSQUE on the entry and, according to its value, proceed to different parts of his program. 
The interrupt and resume sections of a SHRIVE routine see to the preservation (interrupt) and restoration (resume) of any information (outside of arrays JINN and ANNALS) required to resume execution. The JINN and ANNALS arrays are automatically cared for by the main program and the SHRIVE routine need not provide for their handling.

This provision for the handling of JINN makes it desirable that the programmer use the unused portion of JINN (through suitable EQUIVALENCE statements) as the repository for information whose restoration would be desired. Since most interrupt-resume functions a re handled by the main program, these sections of SHRIVE may be in many cases, dummy sections that cause immediate return to the main program.

\section{E. Example of a SHRIVE Routine: SHRILL.}

A particular SHRIVE routine is the SHRILL version which supervises the keeping of a library of FORTRAN source cards. The routine sees to functions that permit the library tape to be used as an input tape for a FORTRAN compilation. Further, it permits removal of decks simply by giving their names when deck is preceded in the old library by a card of a format recognized by SHRILL.

Let us describe SHRILL, then, in an orderly fashion. First, SHRILL assumes that it will be given, as an old library, a tape of records converted from a library of FORTRAN source cards made up of single FORTRAN (or MAP) source decks. Each source deck is preceded by a name card, called a JANUS card, which gives the name of the deck following and marks the end of the previous deck. The format of the JANUS card is specified by the control cards, and if one or more programs are desired from the old library, they can be requested by name.

During execution, SHRILL examines each record to see if it is a JANUS record. If it is, SHRILL examines its file of requests, stored in CERES, to see if the corresponding deck is to be written on the new library. All the options of the SHRILL routine are regulated by LUCRE (1):

$$
\operatorname{LUCRE}(1)=\mathrm{L} 1+2 \times \mathrm{L} 2+4 \times \mathrm{L} 4+8 \times \mathrm{L} 8+16 \times \mathrm{L} 16 \text {, }
$$

where $L_{1}$ is $(0,1)$ if each deck of the old library is to be written on the new library (unless, only if) the deck is named explicitly in CERES.

$$
(\mathrm{L} 2, \mathrm{~L} 4, \mathrm{~L} 8, \mathrm{~L} 16)=(0,1) \text { if }(\mathrm{a}, \mathrm{no}) \text { (I. D, JOB, END PASS, END RUN). }
$$

card is to be written on the new library. In the event these cards are to be written, their contents will be taken from locations ( 21 through 32,41 through 52,61 through 72, 81 through 92) of the GUISE array. Locations 1 through 12 of the GUISE array contain the contents of the JANUS card. Some of the words on the JANUS card will not agree with GUISE(1) through GUISE(12), since they must contain the name of the particular deck being introduced. In particular, words 6 through 10 (columns 31 through 60 on the card itself) contain the 30 identifying characters that will be considered the name of the deck. All other characters (columns 1 through 30, 61 through 72 ) must agree precisely with those in words 1 through 5, 11 through 12 on the standard JANUS card stored in. GUISE (1 through 12).

A complete set of control cards, including those needed for SHRILL, is reproduced on the following pages. 


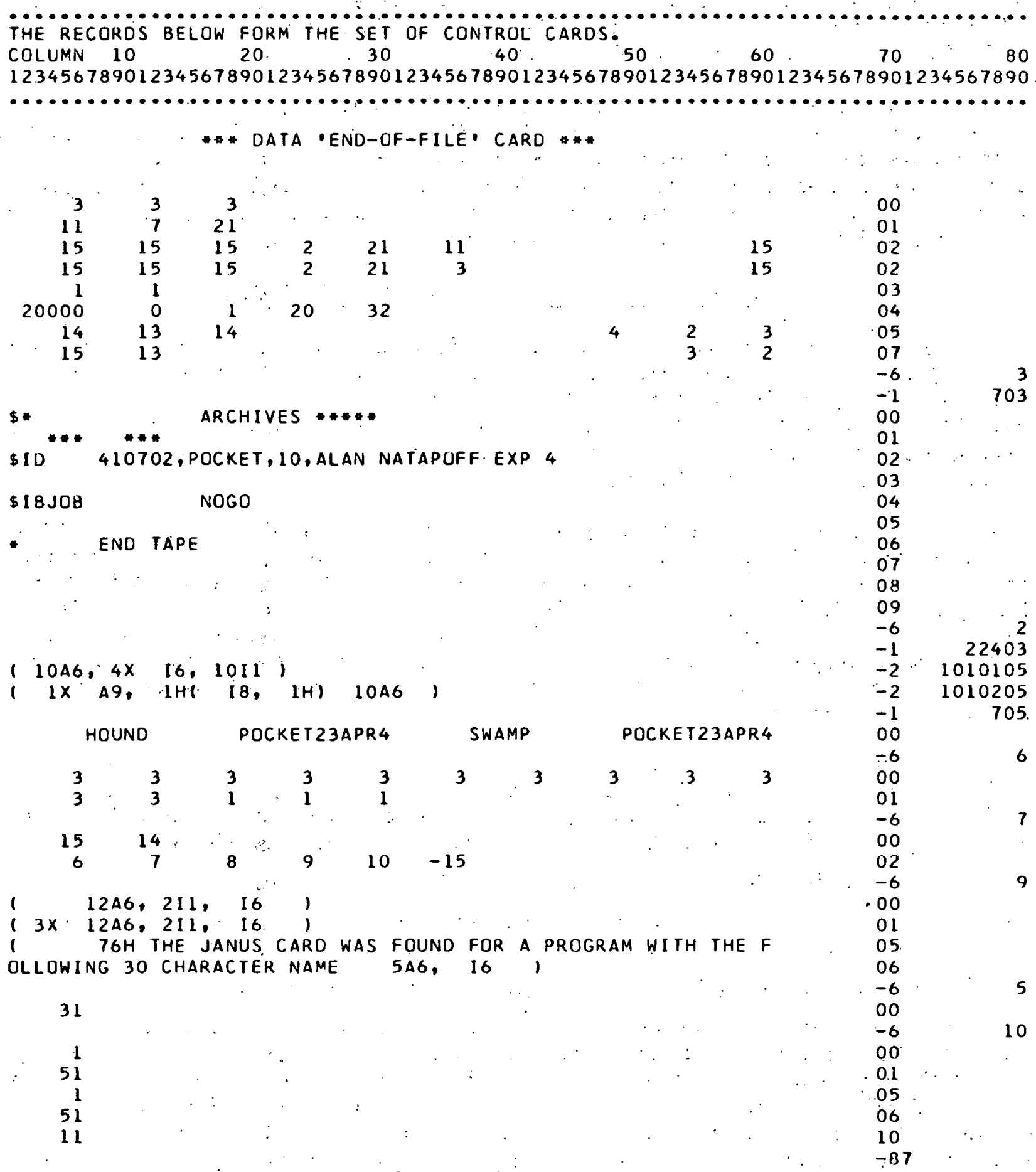




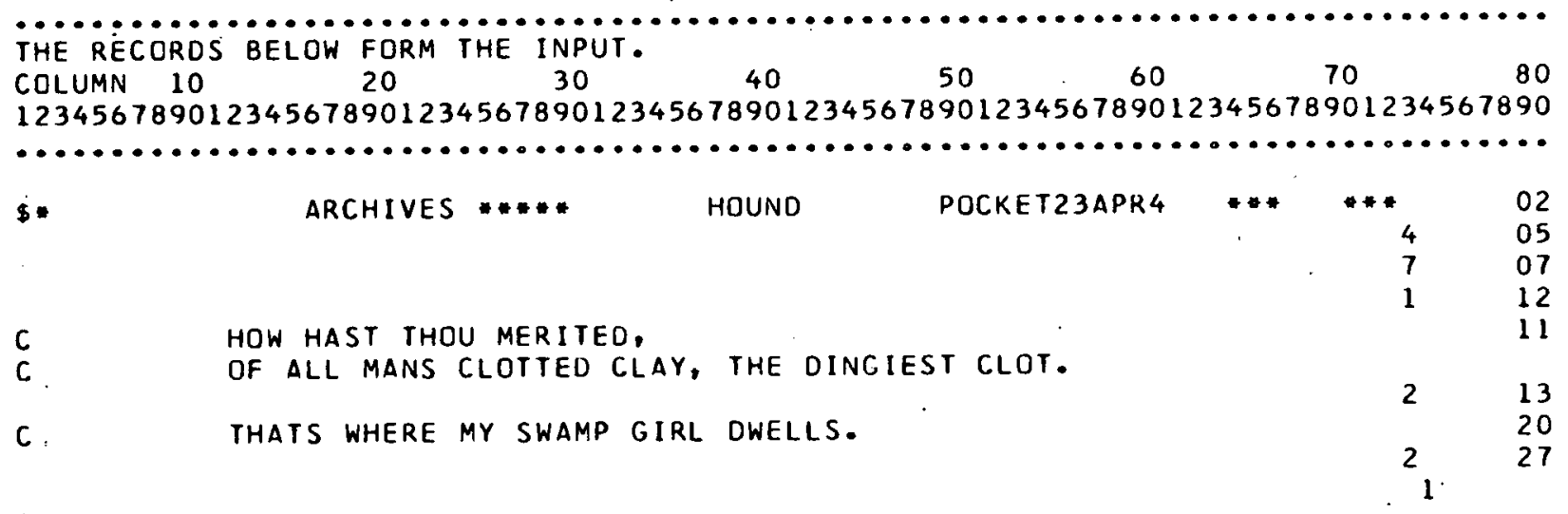




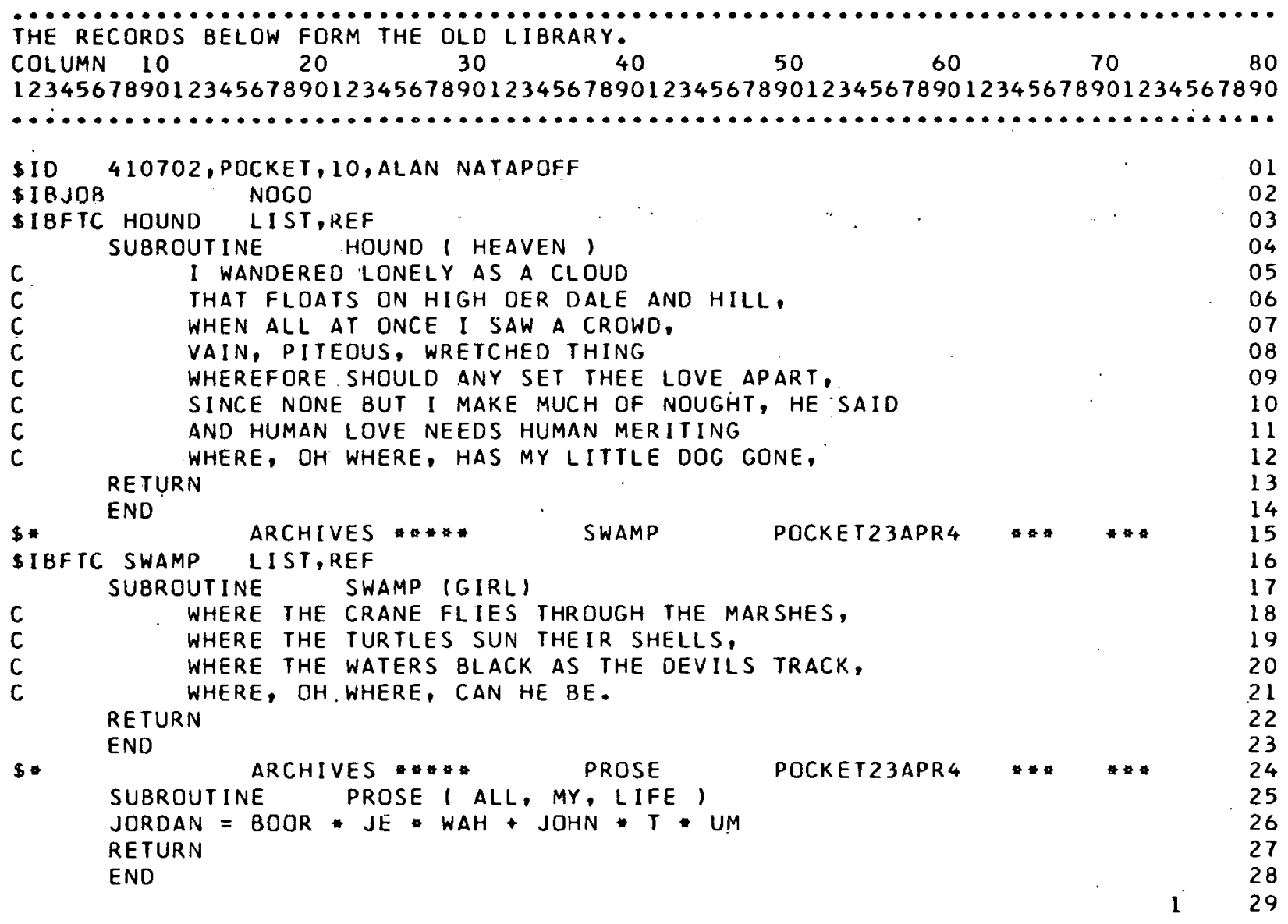




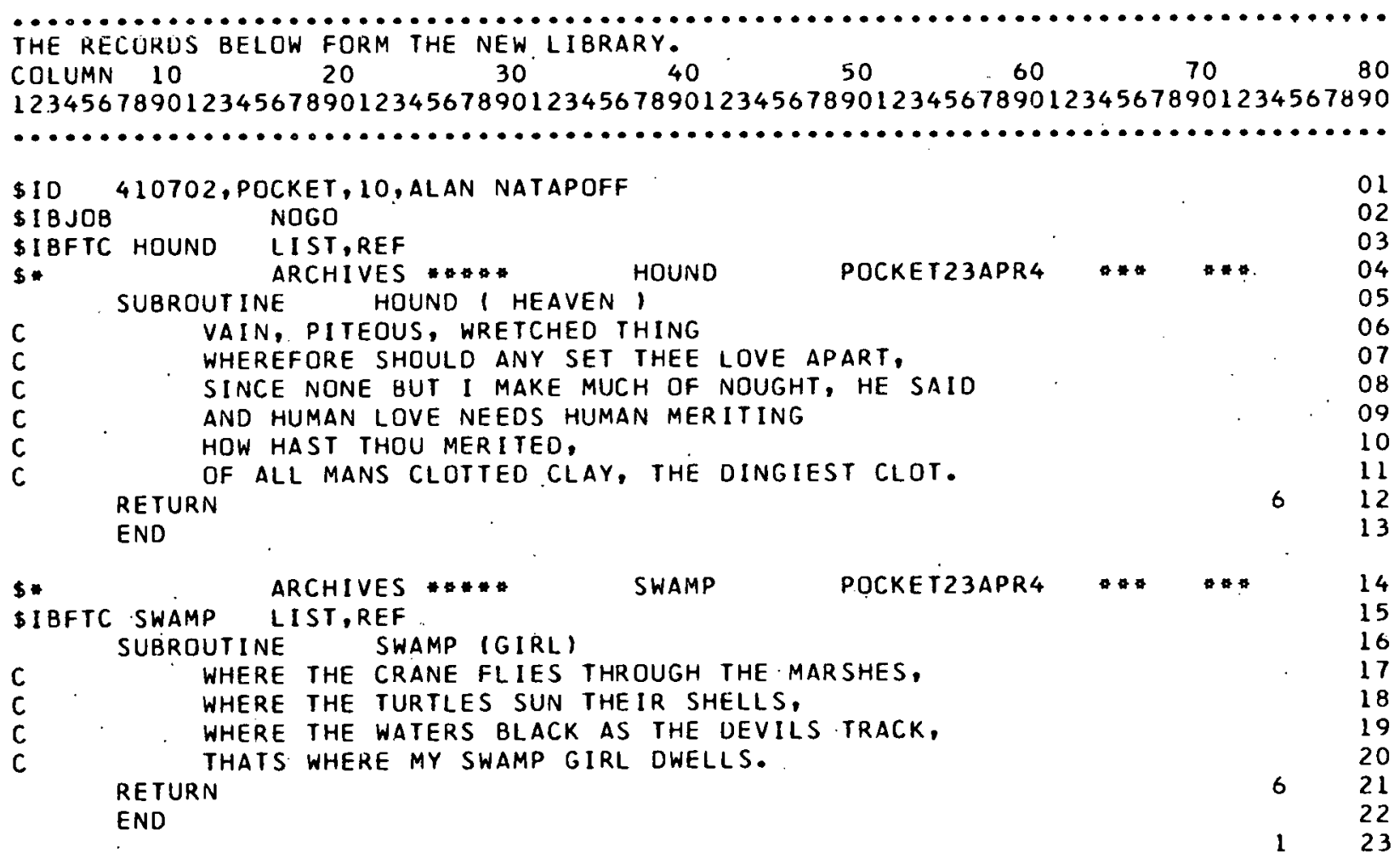


The control cards start with the card after the card saying "-DATA" and end with the card with -87 in columns 69 through 71 . In the IBSYS Monitor for the 7094, it is customary to place a card with a 7-8 punch before any such control cards if they are to be read from the same tape unit as the one from which the converted binary deck is to be read. Similarly, the card with the 7-8 punch in column 1 follows the last card to be read from the tape for the job.

The first group of cards (until the card numbered $\mathrm{N}=-6$ in columns 69 through 70 ) refers to the array JINN in common storage. The first card $(N=0)$ fills in locations JINN (1 through 3) with 3 and JINN ( 4 through 10) with 0 . The 3's indicate that offline comments, record reports on records from the input, and record reports on records from the old library are written on logical tape number 3 .

The card numbered 1 fills JINN(11) through JINN(20), the part of JINN known as "MUSSET" which is used to guide the BARD routine. The first word on this card indicates that the 11th location of each section of LOB.OS refers to the format to be used by the BARD routine. In particular, LOBOS(11) contains the address of the first word of the format to be used in writing record reports on input records. Similarly, we have $\operatorname{LOBOS}(11+\mathrm{MACRO})=\operatorname{LOBOS}(61)$, since $\mathrm{MACRO}=50$ (s ee BLEDA-CIT subroutines) refers to the first word of the format to be used in writing record reports on records from the old library. In our particular case we have $\operatorname{LOBOS}(11)=\operatorname{LOBOS}(61)=51$ (as we shall see later), which implies that the format used for reports on records from both sources is that stored starting at FORM(51). SHRILL suppresses reports for all records except JANUS by setting LAW $=3$. When a JANUS record is encountered, SHRILL sets LAW equal to zero, permitting a record report to be written by BARD.

The second word on card number 1 indicates that the list of words to be written out with the format specified above is stored in block 7 (JORDAN). We store it there because JORDAN has 1024 words assigned to it (see BLEDA-CIT) and uses only 2 of these for the current application. The third word indicates that this list begins in the 21 st location of JORDAN. Looking at card 2 of the JORDAN block, we find that the list of fields to be written out with the format specified is $(6,7,8,9,10,-15)$, which means that fields $6,7,8,9,10$, and 15 will be written out. The minus sign on the 15 indicates that it is the last number of the list.

The first three fields of card 2 ("LASER") give the number of fields (15) appearing on a record of the input, old library, and new library, respectively.

There are two successive cards with $\mathrm{N}=2$, implying that the first will be written over by the second. This is sometimes a convenience. When a given set of data is used often and one parameter is sometimes changed, appropriate cards with both parameters may be kept together and permitted on any given run to cause the desired parameter to be used. In our case, we write the new library on logical tape 11 for ordinary runs and on logical tape 3 (which is the standard monitor system off-line printing tape unit) for test runs. 
In this example, then, the input is read from logical tape 2 , the old library read from 21, and the new library written on 3. The last element (columns 54 through 60) specifies that the records on the new library are to be numbered in field 15.

Card 3 specifies that one input tape reel and one old library tape reel are expected.

Card 4 [JINN (41-50)] states that at most 20,000 records are to be written on each reel of the new library; no records are to be escalated; $1 / 20$ of available storage is to be preserved for escalated records (since we are not escalating, we really need no storage for this purpose); 32 locations are to be set aside for each record in storage (really only 15 are needed).

Card number 5 [JINN (51-60)] specifies that the end-of-reel mark will appear in the 14th field of the record; the command, in the 13th field; and the suppress marks, in the 14th field. (Note that the suppress and end-of-reel marks can share the same field. The end-of-reel mark for the standard version of TENDRE is simply an odd number in the appropriate field. The remaining binary digits in the word may be used to indicate suppression). The actual location of the 14th field on the input card is determined by the format specified by the control cards for the input. In our present case, that format is $(12 A 6,2 I 1, I 6)$, which implies that the 14 th field is composed entirely of column 74 of the input card. If that column contains an odd digit, TENDRE regards the card containing it as marking the end of the reel. The card itself is ignored otherwise. See the listing of subroutine TENDRE for further details.

The last three fields on the card specify that the suppression base is 4 , the suppression divisor is 2 , and the suppression insertion is 3 . Thus, if a record is suppressed, as record 27 is in the set of cards below, one obtains the value of the 14 th field (0), divided by the suppress divisor (2). The first remaining digit to the base 4 is set to 3 , and the result is multiplied back by 2 . Thus the result of the suppression is that the 14th field of the suppressed record is changed from 0 to 6.

Card 7 fills JINN (71) through JINN (80), a region called "LOREN" in the program. It specifies which pieces of information on an edit record are to be kept in storage. The information is used by the CENSOR routine. This particular card tells the program that words number 15 and 13 are to be saved in the first and second words in storage assigned to this edit reference. Word number 15 of every record contains the record number, and word number 13, the command. Thus in the memory we have the name of the record referred to and the edit command that is to be applied to it. The 2 appearing in the last field of this control card indicates that only two pieces of information are to be extracted from each edit record. The 3 in the nextto-last field indicates that three spaces are to be left in storage for each edit record. The extra location is used to indicate whether the editing specified by the record has been carried out.

This completes the specification of the JINN array. The card numbered $N=-6$ indicates the end of the group. The 3 in column 80 of that card indicates that the next array to be filled i.s. GUISE, which is assumed to be filled by records of E-type format. Card $N=-1$ indicates that the format type is to be 
changed to 3 (Alphameric) as specified by columns 79 through 80 , the new mode is to be 7 (columns 76 through 78 ), and the length of the array GUISE is to remain unchanged, since the replacement-value columns 71 through 75 , is zero.

This change of format facilitates the reading in of the basic formats for (JANUS, I. D., JOB, END PASS, END RUN) cards, respectively, in GUISE (1 through 12, 21 through 32, 41 through 52, 61 through 72, and 81 through 92), as discussed above:

The following five pairs of cards contain the forms for the five kinds of cards mentioned. For example, cards numbered 2 and 3 , which are read into GUISE (21 through 30) and (31 through 40), contain the form of the ID card to be written on the front of the new library (unless suppressed). This permits the new library to be used as a direct input to the FORTRAN compiler through the IBSYS Monitor system. Note that a blank record is written at the end of the run.

The array GUISE is ended by the card numbered -6 , which indicates (columns 79 and $80=02$ ) that the next array to be read is array number 2 , CERES. The program assumes that CERES is to be filled by cards in type -2 (E) format. The following card, numbered -1 , specifies that the mode is to be changed to 224 and that records of type 3 are to follow (instead of type 2).

The two cards numbered -2 that follow illustrate the use of the formatinsertion option of the SPADE'subroutine. There is no need in this case for the operations they perform, since the formats they insert to correspond to index $=5$ are already present as index $=3$. For illustrative purposes, we duplicate. The first of the cards inserts the format (10A6, 4X I6, 10I1) in the region appropriate for reading index-5-type control cards. The second inserts the corresponding writing format. The numbers 1010105 and 1010205 in the last columns of these cards break up as 10/10/1/05 and 10/10/2/05, which are interpreted as (NMASK/NFIELD/K/INDEX), where NMASK is the number of fields in the mask, and NFIELD is the number of data fields on a control card of type INDEX. We have $K=(1,2)$ according as the format specified in the data fields of the current control card specify the (reading, writing) format for the INDEXth type of control card. In the present case, we have NMASK $=$ NFIELD $=1 \overline{0}$ and INDEX $=5$. There is some redundancy in that NMASK and NFIELD are specified twice, once on each of the two cards. The last specification survives. The format so inserted remains until another format is inserted in its place.

The next card, numbered -1, changes the mode to 7 and the index to 5 . That is, the program now expects control cards prepared according to the formats just read in for index 5 . This is necessary and convenient for reading in the $B C D$ information to follow.

For the SHRILL version of SHRIVE, CERES contains the names of programs (five computer words per name) in the old library that are to be processed by the POCKET library. In the given case, there are two such program decks in the library identified by the two 30 characters names: 
Each such name is arbitrary, but must match precisely the 30 variable characters on the JANUS card of the deck being referred to. The names we construct have five BCD words of which the first is currently blank, the second gives the name used to refer to the deck in calling sequences, the third gives. a version name if there are several versions of the deck; the fourth gives the name of the large system of programs, if any, with which the deck is associ:ated, and the last word is the date of the first compilation or assembly of the deck.

The references to CERES end with the card numbered -6, which also specifies that the next array to be referred to is the sixth, KHYYBER, which refers to the form of each element of the record and is assumed to be filled by records in integer form (format number 1).

The cards in our example specify that the first 12 fields (six characters each) of our record have BCD (3) form and the next three, integer form (1). That is, the first 72 columns contain the data to be compiled and the last eight columns contain integers used by the program (end-of-reel mark, suppress mark, command, record number). The next "-6" card specifies that the seventh array (JORDAN) will be filled next. JORDAN specifies that records are to be ordered according to their 15th fields; if these are the same, then by their 14th fields. The information on card 2 refers to BARD and is dis cussed above in the paragraphs on the JINN section.

Card -6 specifies that the array FORM is next to be filled. Two are format statements stored in FORM, one starting in FORM(1), the other in FORM(11). These format's are similar, but both are used. The first format is that of the tape converted from FORTRAN source cards. Our reading uses this format. The second format is used for writing where the output tape is to be'printed using the standard-carriage tape. Were the same format used for both, the print program would interpret the first character of each record as a carriage-control character. The first character would then be lost, and the printing would be chaotic whenever the first character of the record was not a blank. The third format is used with BARD and is discussed above with the paragraphs on JINN.

The next card numbered -6 indicates that the 5 th array, LUCRE, is to be filled next. LUCRE(1) $=31$ implies that the only programs to be written on the new library are the two named in CERES (HOUND, SWAMP). Further, there is no ID, JOB, END PASS, and END RUN record written on the new library during the current run.

The last array, LOBOS (the tenth array), contains $\operatorname{LOBOS}(1)=1=\operatorname{LOBOS}(51)$, and $\operatorname{LOBOS}(101)=11$. These numbers give (since each input record corresponds to a single record in memory for the current run) the locations in array FORM of the format to be used for each source. In particular, LOBOS [(I-1) XMACRO+1] contains the location in FORM of the starting point of the format to be used with the records of the Ith source. Specifically, since MACRO is 50 (set by BLEDA-CIT) for the version of POCKET we are describing, the starting point for the (input, old library, new library) format is contained in [LOBOS(1), LOBOS(51), LOBOS(101)]:. The (input, old library, new library) format, therefore, is stored starting at $[\operatorname{FORM}(1), \operatorname{FORM}(1), \operatorname{FORM}(11)]$ and is 
$[(12 \mathrm{~A} 6,2 \mathrm{I} 1, \mathrm{I} 6),(12 \mathrm{~A} 6,2 \mathrm{I} 1, \mathrm{I} 6),(3 \mathrm{X} 12 \mathrm{~A} 6,2 \mathrm{I} 1, \mathrm{I} 6)]$. The two $51^{\prime}$ 's refer to BARD. See the paragraphs above on the JINN section.

Card - 8 implies that we have reached the end of the control cards for the pass and that there are no further passes ( -7 would imply only the end of the control cards for the pass).

The 7000000000 in the last ten columns indicates ISSW(1) $=7$ and ISSW $(J)=0$ for all other values of J from 2 to 10 . ISSW(1) $=7$ implies that we have input as well as an old library to read, and that we will write a new library. See the section on control cards for a description of the options implied by the zeros in ISSW(2) through ISSW(10).

In the accompanying figures we have given a sample old library, a sample set of insertions and editing commands (input), and the new library that POCKET produces from these with the given control cards. Let us review a point before we continue. The control cardsare cards that prescribe the library functions POCKET is to perform. They are of a form prescribed by BLEDA-CIT and (for special cases of data of a peculiar form needed for the SHRIVE routine) by the user through the format insertion option of SPADE. The library and input records are not of fixed forms. Their forms are always specified in the control cards by the user. The example given above illustrate this point. The old and new libraries and the input end with records having odd numbers in column 74, a sign recognized by the standard TENDRE routine. The records on the old library (produced by an earlier run of POCKET) are numbered in columns 75 through 80 . The input prescribes that we enter its first record into the library after the record of the old library numbered 2. The second and third records of the input prescribe that we delete the records 5 through 7 on the old library. The fourth record prescribes the deletion of record number 12 of the old library. The next record is to be inserted after record number 11 of the old library. The record following, reading "C OF ALL MANS CLOTTED CLAY THE DINGIEST CLOT" will follow the previous record. We should explain this. This record is blank in the numbering field, which means that its number is interpreted as 0 . The program, after having written record number 11 of the old library and the previous record "C HOW HAST THOU MERITED," must decide whether to write record number 12 of the old library or the record in question, numbered 0, next on the new library: Since zero is less than 12, the input record is written next. This illustrates a convenient means of inserting a series of records into the library.

The two records having 2 in column 73 cause suppression of records 13 and 27 of the old library. This suppression is discussed above. The last record contains an odd number in column 74, indicating the end of the reel.

The new library shows the changes prescribed by the input. 
E. Relaxations Permitted in Restrictions on Form of

Libraries and Input

Any relaxation is permitted that can be confined to the beginning or end of a library or input set in such a way as to permit handling by the SHRIVE routine in its initialization or terminal mode. For example, if the first record of the library is a tape label of format different from those of the records of the library, the SHRIVE routine itself can read the label during its initialization entry and thus leave the remainder of the library as a set of records conforming to the restrictions on uniformity of record. 


\section{SUBROUTINES BLEDA AND CIT}

At the very start of execution, it is desirable to clear certain storage locations and to initialize others with data that do not vary from application to application. These functions are handled by subroutines in order that the main program may be free of the details and that the system may be easily changed from machine to machine.

Some FORTRAN systems have available a BLOCK DATA subroutine facility with which one can load parameters directly into COMMON. Where such a facility exists, such as on the 7094, the system uses such a BLOCK DATA subroutine and calls it BLEDA. Those initializations not conveniently performed in BLEDA are performed by CIT, a subroutine that is called once at the very start of execution. In those systems lacking a BLOCK DATA subroutine facility, the whole burden of initialization is borne by CI'T.

The initialization embraces formats and parameters used by SPADE, allocation parameters for the STRATE array (a large array broken up into smaller ones by these parameters), and parameters describing the machine and system. It also clears the large regions in storage STRATE and ANNALS. For further information, refer to the appropriate listing and the table given below of the partitioning of the STRATE array:

\begin{tabular}{|c|c|c|c|c|}
\hline \multicolumn{2}{|c|}{ Name of Ith array } & \multirow{2}{*}{$\frac{\text { IF }}{\text { INDIES }(I)}$} & \multirow{2}{*}{$\frac{\text { LOC }}{\text { LESTER (I) }}$} & \multirow{2}{*}{$\begin{array}{c}\text { LONG } \\
\text { MALICE (I) }\end{array}$} \\
\hline 1 & $\operatorname{ADAM}(I)$ & & & \\
\hline 1 & JINN & 1 & 0 & 1000 \\
\hline 2 & CERES & 2 & 1000 & 400 \\
\hline 3 & GUISE & 2 & 1400 & 100 \\
\hline 4 & ICARUS & 1 & 1500 & 30 \\
\hline 5 & LUCRE & 1 & 1530 & 20 \\
\hline 6 & KHYBER & 1 & 1550 & 1024 \\
\hline 7 & JORDAN & 1 & 2574 & 1024 \\
\hline 8 & EREWON & 2 & 3600 & 100 \\
\hline 9 & FORM & 3 & 3700 & 150 \\
\hline 10 & LOBOS & 1 & 3850 & 150 \\
\hline 11 & MAFIA & 1 & 4000 & 150 \\
\hline 12 & INGRAM & 1 & 4150 & 150 \\
\hline
\end{tabular}

The array name is merely a label assigned to a portion of mernory in the STRATE block. It is a set of six characters printed out when the array is listed by SPADE. 
IF is an indicator telling what format of control card is assumed to be appropriate for filling the array. The user may change this assumption with an appropriate control card numbered -1 , as described in the section on the SPADE routine. IF $=(1,2,3,4)$ corresponds to $(I, E, A, O)$ type control cards.

LOC is the location of the last word in STRATE before the beginning of the block. For example, for array LUCRE, we have LOC = 1530 in the standard version of BLEDA-CIT. This means that the first word of LUCRE is STRATE. (1531).

LONG is the number of words of storage set aside in STRATE for the array. 
VII. ESCALATION

For comparison of neighboring records or integration of several records, it is often convenient to save records after they have been written on the new library. If NUECES (a parameter in JINN) is set equal to $N$, then, in addition to the records being processed, the $\mathrm{N}$ most recently processed records will be stored in the ANNALS. After each new record is processed, it is inserted in the top of the file of "saved" records, and each record currently there is dropped one record lower. The former Nth record is lost. The processing of the record should be indicated on the record after the last location used (LESBOS) but before the last location that is escalated (NIBBLE). This is to distinguish records that have been deleted. If there are, for example, 155 words used in a record, perhaps 160 would be specified as NIBBLE, and 160 locations would thereafter be moved as a unit with each record. One could then insert additional pieces of information in locations 156 through 160 and have them available for later comparisons. In particular, the COMMON parameter MIRAGE summarizes the previous handling of a record. The SHRIVE routine could insert MIRAGE in the 156 th location of the previous record (since, at the time of entry into SHRIVE, MIRAGE refers to the previous record. MIRAGE is calculated after exit from SHRIVE), and thereby have the necessary processing information available. In particular, in the mentioned case, we could insert MIRAGE in the 316 th location of the record being processed in SHRIVE (i.e. 160 locations for the current record +156 locations into the next record which, when escalation is performed, is next in storage).

Comparisons of records stored in memory are facilitated by the RIVAL routine, whose operation is described in its Fortran listing. 


\title{
VIII. RECOMMENDED PROCEDURES FOR SETTING UP
}

\author{
A Library Using the POCKET System
}

The POCKET library system is designed for use with relatively small or infrequently referenced libraries. Large libraries referred to often will usually. repay the effort needed to produce a fast, specialized system. POCKET could be used in the interim, however. A large library is one that costs a lot to run through once. Each user must decide for himself whether his library is large. Ordinarily, libraries of 20,000 or fewer records would be considered small.

Having said something about the number of records in a library, we should say something about the length of a record. It is usual to enter new information into a library by punching it onto one or more cards, then converting the cards to a tape and reading the tape. Therefore, we define a short record as one that can be entered on a single card, and a. long record as one that requires more than one card but contains fewer than 1024 fields of information. Records that contain 1024 words or more are considered very long. As presently written, POCKET is definitely unsuitable for handling very long records. The prociedure recommended for short and long records differ in some respects. In one respect they are similar. Since the input and output of $\mathrm{BCD}$ records requires execution of a conversion, it is more economical to have one's library written in binary form, whatever the form of the input.

If the records are long, it is well to leave blank much of the first card of each input record (and hence the first few fields of each record are uncommitted) to accommodate various fields to be used by the library system. Unless one writes a special TENDRE subroutine that will recognize the end of his input and old library reels, one field must be set aside for the end-ofreel mark if the appropriate field contains an odd number. In addition, a field must be set aside for the command. Also one field must be set aside for numbering the records, and up to four fields for the optional indirect suppression features described in the section on the CENSOR routine. Lastly at least one field must be provided for suppression marks if any are to be used. The end-of-reel field may be made to do double duty, however. Thus, a minimum of three and a provisional maximum of eight or nine integer fields must be left fallow to permit the use of POCKET's optional features.

On:a.long record, the first card (card 0) may be left to these tasks with data beginning on card 1. On a short record, such as the one in our illustration in the Section on SHRIVE, there is usually not enough room for such prodigality. In our example, we left 1 field (column 73 ) for the command, 1 field (column 74) for the end-of-reel mark and suppression, and 1 field (columns 75 through 80 ) for numbering the: records. 


\section{ACKNOW LEDGMENTS}

The author'would like to apologize to other lovers of the work of Franics Thompson for paraphrasing his famous poem for use as an example in the text. Extensive direct quotation was avoided to simplify copyright conside rations.

It is a pleasure to acknowledge the contributions of Mark W...Horovitz and Jerry Borges of the Lawrence Radiation Laboratory Mathematics and Computing Group. Their critical suggestions helped in the devclopment of the program itself and of: its documentation. Their contributions and those of Albert Briggs, now of the Peace Corps; helped extend the scope and : enhance the utility of the result.

This work was performed under the auspices of the U. S. Atomic Energy Commission. 
This report was prepared as an account of Government sponsored work. Neither the United States, nor the Commission, nor any person acting on behalf of the Commission:

A. Makes any warranty or representation, expressed or implied, with respect to the accuracy, completeness, or usefulness of the information contained in this report, or that the use of any information, apparatus, method, or process disclosed in this report may not infringe privately owned rights; or

B. Assumes any liabilities with respect to the use of, or for damages resulting from the use of any information, apparatus, method, or process disclosed in this report.

As used in the above, "person acting on behalf of the Commission" includes any employee or contractor of the Commission, or employee of such contractor, to the extent that such employee or contractor of the Commission, or employee of such contractor prepares, disseminates, or provides access to, any information pursuant to his employment or contract with the Commission, or his employment with such contractor. 